\title{
On the Verifiability of Evolutionary Psychological Theories: An Analysis of the Psychology of Scientific Persuasion
}

\author{
Lucian Gideon Conway, III \\ Department of Psychology \\ Indiana State University \\ Mark Schaller \\ Department of Psychology \\ University of British Columbia
}

\begin{abstract}
Evolutionary psychological theories have engendered much skepticism in the modern scientific climate. Why? We argue that, although sometimes couched in the language of unfalsifiability, the skepticism results primarily from the perception that evolutionary theories are less verifiable than traditional psychological theories. It is more difficult to be convinced of the veracity of an evolutionary psychological theory because an additional layer of inference must be logically traversed: One not only has to be persuaded that a particular model of contemporary psychological processes uniquely predicts observed phenomena, one must also be persuaded that a model of deeply historical processes uniquely predicts the model of psychological processes. This analysis of the psychology of scientific persuasion yields a number of specific suggestions for the development, testing, and discussion of evolutionary psychological theories.
\end{abstract}

New theoretical perspectives in science are not born easily. The broad notions and odd angles of novel ideas move haltingly through cognitive passageways that have been worn to the size and shape of conventional wisdom. This difficult birth can be followed by a painful childhood. Novel perspectives almost always meet some sort of skeptical resistance, and even the most useful theoretical ideas must sometimes-like new kids in school-endure harsh challenges and critical rebukes before they are eventually accepted on the intellectual playground.

This perspective is worth keeping in mind when considering the recent emergence of evolutionary psychology - the metatheoretical perspective that applies evolutionary biological thinking to psychological phenomena. Not surprisingly, evolutionary psychology has precipitated a considerable amount of scholarly debate. During the relatively short period of time that evolutionary thinking has existed on the landscape of the psychological sciences, it has been enthusiastically criticized and has been defended with equal ardor. Although many complex issues are obscured by the sound and fury of

The writing of this article was partially supported by a grant to Mark Schaller from the Social Sciences and Humanities Research Council of Canada. The authors thank Doug Kenrick and Virgil Sheets for helpful comments on a prior draft of this article.

Requests for reprints should be sent to Lucian Gideon Conway, III, Department of Psychology, Root Hall, Indiana State University, Terre Haute, IN 47809. E-mail: pyconway@ scifac.indstate.edu these scientific squabbles, one thing is clear: Evolutionary psychological perspectives are difficult for many psychologists to swallow whole.

Why is this? Critics of evolutionary psychology might argue that the reasons lie in the contents of evolutionary thinking. Proponents of evolutionary psychology may be tempted to believe that the reasons lie in the heads of the critics. Neither reaction is entirely true, nor entirely false. When one separates wheat from chaff in the harvest of criticisms and rebuttals, it becomes apparent that evolutionary psychological theories have a different logical structure than most other psychological theories and that these differences in logical structure have important influences on the psychological processes that guide scientists' appraisals of the theories. Our purpose in this article is to examine this subtle interaction between the logic of evolutionary psychology and the psychologic of scientists.

In doing so, our goals are twofold. At a broad, general level, we hope to illuminate the often overlooked but fundamental role that psychological inference and persuasion processes play in determining scientists' evaluative impressions of any hypothesis or theory in the psychological sciences. More specifically, we attempt to apply this set of principles constructively to the case of evolutionary psychology. We summarize implications specific to evolutionary psychological theories in the hope that theories within this domain might be articulated in such a way that they more fully — and less painfully - realize their potential. The 
considerations presented here are relevant not only to the debate about evolutionary psychological theories in general but also to the social psychological community in particular. Given the logical necessity of reproduction to the passing on of one's genes, it is perhaps unsurprising that much attention within evolutionary psychology has been paid to human mating behavior (e.g., Buss, Haselton, Shackelford, Bleske, \& Wakefield, 1998). This focus on such an obviously social phenomenon has put social psychological research on or near center stage for much of the debate relevant to evolutionary psychological theories. Thus, although the ideas described in this approach are applicable beyond the walls of social psychology, in choosing illustrative examples we have kept our focus on theories that are decidedly social in nature.

\section{Scope of This Article}

In this article, we attempt to identify one particular set of reasons why people are skeptical of evolutionary theories. That set of reasons focuses on the logic of inference that is applied to specific theories and the subsequent impact of that logic on the psychology of scientists. We recognize, however, that many processes outside of those that we discuss also impact the persuasiveness of evolutionary psychological theories. For example, scientists think the probability that a particular metatheoretical perspective will yield a true theory or hypothesis is greatly influenced by the perception that the perspective has previously yielded true hypotheses. Thus, there is a cumulative effect of positive results for a given theoretical perspective: To the degree that scientists perceive that evolutionary psychological theories in general have had success, they are more likely to perceive specific new evolutionary psychological theories to be true (see Ketelaar \& Ellis, 2000). However, these and other processes, although important, fall beyond the scope of this article.

\section{What Do Scientists Want?}

To understand the processes underlying the evaluation of theories in evolutionary psychology (and theories in other domains as well), it is necessary to consider the context within which those theories are evaluated. This context is defined by the goals of scientists (Crandall \& Schaller, in press; Kitcher, 1993; Kruglanski, 1994). Scientists seek to discover the facts of reality, to provide true explanations for those facts, and to use those explanations to accurately predict new facts. Given these goals, scientists value veracity-in the form of empirical certification-above all else (Merton, 1942). In fact, among many philosophers of science, the term knowledge itself implies veracity (Bechtel, 1988).

Given this fundamental value, scientists are pragmatic in choosing the standards against which theories are judged. The gold standard is the extent to which the statements in theory provide accurate representations of reality. First and foremost, we ask this question about any theory: "Is it true?"

\section{Issues of Falsifiability and Falsification}

The positivist philosophy that guides psychological science informs us that we can never answer this question-"Is it true?"-in the affirmative, only in the negative (Popper, 1959). We can never claim with $100 \%$ certainty that a theory is true, but we can sometimes confidently reject a theory as false. It is for this reason that falsifiability is a necessary feature of any scientific theory. Nonfalsifiable statements may well be true, of course, but observations of reality are not diagnostic of their truth. Consequently, for individuals whose goal is the determination of truth, nonfalsifiable statements are-for good reason—dismissed as scientifically irrelevant.

\section{Falsifiability of Evolutionary Psychological Theories}

There is a long history of lodging charges of nonfalsifiability against evolutionary thinking in the biological sciences (Peters, 1976; Popper, 1959). The same charges have been leveled against applications of evolutionary thinking in the psychological sciences as well (e.g., Gould, 1997; Kitcher, 1985). Are these charges valid? Are evolutionary psychological theories nonfalsifiable? No. As others have taken pains to articulate (e.g., Kenrick \& Simpson, 1997; Ketelaar \& Ellis, 2000), theories and hypotheses developed within evolutionary psychological frameworks are exactly as falsifiable as any other theory or hypothesis in psychology.

To illustrate, consider two different theoretical structures within psychology. The first is an evolutionary psychological model specifying the evolutionary pressures attendant on differential parental investment in childbirth within ancient populations (e.g., Buss \& Schmidt, 1993). This model suggests that in ancient environments it would have been advantageous (with respect to gene survival) for men to focus on finding mates that were maximally fertile, whereas it would have been advantageous for women to focus on finding mates that were best able to provide resources for their children. The model further suggests that, because of these differential selection pressures, men would have developed a different set of psychological cues for mate preferences than women: Men would be likely to 
focus on cues relevant to fertility, whereas women would have developed a set of cues relevant to resource provision. The second model is a social structural model specifying different contemporary cultural norms and expectations operating on men and women (e.g., Eagly \& Wood, 1999). This model suggests that, because of discrepancies in gender status, men have adopted dominant social roles, whereas women have adopted (or been subjected to) subordinate social roles. The model further suggests that, given this present state of affairs, it is advantageous for men to seek mates that are best able to fill subordinate social roles such as housekeeping, whereas it is advantageous for women to seek mates that are best able to provide direct resources to them (as they cannot get those resources themselves).

Through the process of logical deduction, both the evolutionary and social structural theories outlined previously yield an identical hypothesis: In general, men prefer younger women for mating partners, whereas women prefer older men. Now suppose that empirical evidence is collected that examines the relation between gender and the preferred age of mating partners, and suppose that this evidence reveals no relation whatsoever. As long as this non-effect cannot be easily attributable to shoddy measurement, it bears negatively on the hypothesis; therefore, it bears negatively on all theoretical structures that logically yield that hypothesis. Just as the model specifying contemporary cultural norms would be empirically falsified by this non-observation, so too-and to an equal extent-would the model specifying evolutionary pressures be empirically falsified. ${ }^{1}$ Clearly, then, skepticism accorded to evolutionary psychological theories does not result from the logic of falsifiability (Schaller \& Conway, 2000). ${ }^{2}$

\footnotetext{
${ }^{1}$ Of course, falsification of a theory is rarely, if ever, based on a single negative result, but rather depends on an accumulation of negative results. It is an inferentially complex passage from an "empirical disconfirmation" to the full falsification of a theory (Greenwald \& Ronis, 1981; Laudan, 1977; Quine, 1953).

${ }^{2}$ The falsifiability charge may partially rest on a confusion about what an evolutionary theory actually is. Consider again the evolutionary theoretical statement that "differential selection pressures for men and women led to differential rates of promiscuity in men and women." As discussed previously, this statement, taken as a whole, is completely falsifiable: If men and women do not have differential promiscuity rates, then it is clear that differential selection pressures did not lead to differential promiscuity rates. However, evidence that men and women do not have differential promiscuity rates does not mean that the hypothesized differential selection pressures did not exist. The key distinction is that evolutionary theories of psychology do not hypothesize that "certain evolutionary pressures existed;" rather, they hypothesize that "certain evolutionary pressures led to certain psychological structures." The first statement cannot be falsified by psychological studies; the second statement can. It is the second statement that evolutionary psychological theories are making.
}

\section{Falsification of Evolutionary Psychological Theories}

Although a theory must be falsifiable to be considered a scientific theory, the falsifiability of a theory itself has almost no bearing whatsoever on the extent to which that theory is judged to be true. Logically (according to the positivist philosophy that most psychological scientists ascribe to), assessments of truth are based not on falsifiability but the results of exposure to falsification: To what extent has a theory survived actual exposure to empirical observations that are diagnostic of that theory's accuracy? If an observation matches the predictions deduced from the theory, then the theory survives that exposure. The more exposures survived, the more the theory is perceived-however tentatively- to be true.

Is it possible that theories in evolutionary psychology are greeted with special skepticism because these theories are-compared to other theories in psychology-underexposed to opportunities for falsification? Perhaps this is true. Given the relative youth of evolutionary psychology, there are a number of novel theories within the domain that have not yet had the opportunity to be extensively tested against empirical observations. For these untested theories, scientists adhering to a positivist approach should logically maintain some skepticism.

On the other hand, this state of affairs is no different from that in any other domain of active psychological inquiry. Although there are some evolutionary psychological theories that are just beginning to be tested empirically, there are others that have survived exposure to numerous opportunities for empirical disconfirmation. Consider, for example, theories based on evolutionary models of differential parental investment (Buss \& Schmidt, 1993). These theories have received by far the most empirical attention of any theoretical structures within the domain of evolutionary psychology and have survived dozens - if not hundreds - of independent exposures to falsification (Buss, 1989; Buss \& Schmidt, 1993; Gangestad \& Simpson, 2000; Kenrick \& Keefe, 1992). Yet, curiously, it is within the context of these theories that charges of nonfalsifiability and other declarations of disbelief are most often aired. Thus, it seems unlikely that underexposure to diagnostic data accounts for the skepticism that chronically greets theories in evolutionary psychology.

\section{Why Does the Skepticism Persist?}

Apparently, something other than the positivist logic of falsification underlies skepticism about theories in evolutionary psychology. So why does the feeling persist that evolutionary psychological theories are 
less falsifiable —or perhaps just less true-than other theories in psychology?

One possibility that is commonly asserted (at least informally) by advocates of evolutionary psychology is that skeptics are merely undereducatedthat they are not yet well informed about either the actual nature of evolutionary psychological theories or about the wealth of diagnostic evidence that supports those theories. This explanation implies that evolutionary psychological theories are no different than any other theory in psychology and that as people become more familiar with the actual structure of evolutionary theories and the considerable empirical literature bearing on those theories, the skepticism feelings will disappear.

This explanation probably has at least some merit; lots of psychologists (and many more people outside the field) remain only vaguely aware of the contents of scientific inquiry within evolutionary psychology. However, we suspect this explanation is incomplete and its implication is overly optimistic.

Close attention to the underlying logical structure of theories in evolutionary psychology reveals that these theories are, in some important ways, different from most other theories in psychology. Moreover, regardless of any ostensible adherence to a positivist philosophy of science, the process through which individuals become persuaded as to the truth of a theory has little to do with the strict logic of falsification (Schaller \& Conway, 2000). By attending to the interaction between the logical structure of evolutionary psychological theories and the psychological processes underlying individuals' assessments of those theories, we can more completely understand the origins of the skepticism that greets evolutionary psychology. This analysis also yields a number of implications and suggestions pertaining to the manner in which evolutionary psychological theories might best be constructed, tested, and discussed.

\section{Inferential Logic of Scientific Persuasion}

Human beings-including scientists-are intuitive verificationists, not intuitive falsificationists. As humans, we do not care very much for what is not; we only really care for what is (Schaller \& Conway, 2000). Scientists are persuaded as to the accuracy of a theory through an intuitively appealing logic: We believe that a theory is true if (a) empirical results match the predictions of the theory and (b) these empirical results cannot obviously be explained otherwise. Scientists' experimental methodologies are fueled primarily by this logic. To the extent that obtained empirical results reflect positively on a focal theory and cannot obviously be explained otherwise, the focal theory is judged (tentatively) to be true. ${ }^{3}$

Of course, as scientists we do not typically compare theories directly to empirical data. We judge the veracity of theories only after first judging the veracity of hypotheses deduced from those theories. To understand the inferential logic through which scientists are persuaded as to the truth of a theory, we must first consider the inferential logic through which we are persuaded as to the truth of a hypothesis.

\section{Perceived Truth of a Hypothesis}

The confidence in the truth of any hypothesis is based on answers to two questions: (a) Just how well do the observed empirical results match up with the conceptual hypothesis? and (b) just how easily can these same empirical results be accounted for by alternative explanations?

The first of those judgments is informed by a variety of distinct factors: patterns of obtained experimental results, effect sizes indicated by those results, construct validity of experimental operations, and so forth. Considered together, these factors yield a subjective judgment. It is possible to imagine this judgment being made on a sort of "batting-average" scale anchored by endpoints 0 (no correspondence) and 1 (perfect correspondence).

The second subjective judgment-presence of viable alternative explanations-is also informed by a variety of distinct considerations: statistical results of null hypothesis testing, internal validity of experimental methods, and the awareness of other logically plausible conceptual hypotheses predicting the same pattern of results. Considered jointly, these factors yield a subjective judgment that also might be represented on a scale anchored by endpoints of 0 (complete absence of alternative explanations) and 1 (certainty that alternative explanations account for the observed results).

This intuitively appealing logic can be formally expressed by a simple linear equation. Let $A$ represent the extent to which empirical results persuade us as to the veracity of the focal conceptual hypothesis. Let $a$ represent the first judgment (correspondence between conceptual predictions and empirical results) and $a^{\prime}$ represent the second judgment (plausibility of alternative explanations).

$$
A=a^{*}\left(1-a^{\prime}\right)
$$

\footnotetext{
${ }^{3}$ This intuitive logic of scientific inference is not dissimilar from the intuitive logic through which we judge the causes of observed events in our everyday lives (Bem, 1972; Kelley, 1967). More generally, the simple model of inferential logic outlined here is consistent with a more sophisticated model of "explanatory coherence" relevant to both scientific and everyday inference (Kunda \& Thagard, 1996; Thagard, 1992).
} 
Thus, the perceived veracity of a focal conceptual hypothesis is the multiplicative function of the perceived empirical support for the hypothesis and the inverse of the perceived plausibility of some alternative explanation(s) for that apparent support. ${ }^{4}$

\section{Perceived Truth of a Theory}

Of course, scientists think not only in terms of single assertions expressed as hypotheses, but also in terms of coherent clusters of assertions in the form of theories. Theories are at least one logical step removed from hypotheses tested by empirical methods. The typical theory in psychology consists of a set of logically connected statements concerning the operations of some set of psychological processes. These theoretical statements imply-through a process of logical deduction - a set of specific hypotheses about the relations between specific variables. Each of these hypotheses is subject to empirical test through the logical process described previously. However, support for a specific conceptual hypothesis does not guarantee the subjective persuasiveness concerning the veracity of the theory from which it is deduced. This is because no conceptual hypothesis can be assumed to be deducible solely from that one theory. It is always possible (and highly likely) that the same conceptual hypotheses might be deduced from one or more different theoretical structures. Thus, given perceived empirical support for a focal hypothesis, the perceived veracity of a theory of psychological processes depends on two judgments: (a) the extent to which that hypothesis is logically deduced from the theory and (b) the extent to which it cannot be logically deduced from alterative theories.

The first of those judgments is based on a subjective assessment of the tightness of the logic through which the hypothesis is deduced from the focal theory. The second of those judgments is based on a subjective assessment of the logical ease through which the same hypothesis is deducible from alternative theories.

For the sake of formal expression, both judgments might be represented on 0 to 1 scales and may be symbolized, respectively, as $b$ and $b^{\prime}$. The extent to which a particular empirical result persuades the observer as to the veracity of a particular theory $(B)$ must take into ac-

\footnotetext{
${ }^{4}$ These simple considerations reveal why falsification plays such a minor role in actual scientific persuasion. A conceptual hypothesis may correspond exactly to a set of empirical results (and therefore resist falsification) but may still be believed with little confidence because of the presence of other, equally plausible, explanations. Similarly, difficulties in operationalizing conceptual variables may make it difficult to empirically falsify a conceptual hypothesis (Quine, 1953), but this does not mean the hypothesis is viewed as true. It is quite the contrary: The same operational difficulties interfere with the ability to obtain support for the hypothesis, and this is what really matters in scientific persuasion.
}

count not just the judgments symbolized by $a$ and $a^{\prime}$ but also these new judgments $b$ and $b^{\prime}$ :

$$
B=\left[a *\left(1-a^{\prime}\right)\right] *\left[\mathrm{~b} *\left(1-b^{\prime}\right)\right]
$$

For most theories in psychology, the logic of inference ends there. That is because most theories in psychology simply specify the operations of psychological processes-the causal relations between more or less contemporaneous variables. Yet some theories go further.

Some theoretical perspectives within psychology articulate not only conceptual models describing the contemporary psychological operations but also articulate models describing the historical origins of those psychological operations. For instance, one social role theory of gender differences identifies a set of long-ago historical origins of contemporary sex-linked divisions of labor (e.g., Eagly \& Wood, 1999). As another example, terror management theory yields many testable hypotheses about contemporary psychological phenomena as a consequence of first articulating a set of historical processes through which cultural norms and self-esteem systems emerged (Greenberg, Solomon \& Pyszczynski, 1997; Solomon, Greenberg, \& Pyszczynski, 1991). Additionally, of course, all theories within evolutionary psychology specify historical models within which the evolutionary origins of contemporary psychological events are described. Evolutionary theories of contemporary human mating behavior (e.g., Buss \& Schmidt, 1993; Gangestad \& Simpson, 2000) not only specify the particular ways in which men and women differ in their approaches to sexual relationships, they also specify the evolutionary mechanisms through which these differences emerged long ago. Evolutionary theories of contemporary human cognition (e.g., Cosmides, 1989; Gigerenzer, 1998) not only specify (just like most other psychological theories) the presence of specific cognitive operations and the effects of contextual cues on those cognitive operations, they also specify (unlike most other psychological theories) the evolutionary mechanisms through which these cognitive systems emerged in the first place, long ago.

Thus, these theories contain two distinct models, not one, and these models address very different types of questions (Conway, 1999; Ketelaar \& Ellis, 2000; Schaller \& Conway, 2000; Simpson \& Gangestad, 2001). Even if one accepts as true the theoretical statements that specify contemporary psychological operations and events, this does not necessarily mean that one accepts as true the statements specifying the historical origins of those operations and events. An additional layer of inference is introduced that bears a structure identical to the two layers of inference summarized previously. Persuasion concerning the veracity of some specific theoretical model of origins is a 
function of two considerations: (a) the extent to which the model of contemporary psychological processes is logically deduced from the historical model of origins and (b) the extent to which it cannot be logically deduced from alternative models specifying distinctly different historical origins.

The first of those judgments is based on a subjective assessment of the soundness of the assumptions and the tightness of the logic through which the model of contemporary psychological events is deduced from the model of historical (e.g., evolutionary) origins. The second of those judgments is based on a subjective assessment of the logical ease through which the same set of psychological events is deducible from alternative models of historical origin.

As mentioned previously, both of these judgments might be represented formally on 0 to 1 scales and symbolized, respectively, as $c$ and $c^{\prime}$. The extent to which a particular empirical result persuades the observer as to the veracity of a particular theory of ultimate origins $(C)$ must take into account not just the judgments symbolized by $a, a^{\prime}, b$, and $b^{\prime}$, but also these new judgments $c$ and $c^{\prime}$ :

$C=\left[a *\left(1-a^{\prime}\right)\right] *\left[b *\left(1-b^{\prime}\right)\right] *\left[c *\left(1-c^{\prime}\right)\right]$.

\section{Summary}

Figure 1 presents schematically the formal logic just outlined. This schematic makes the fundamental point very clear: The more inferentially distal a theoretical model is from empirical results, the more difficult it must be to persuade people that the elements of that model are accurate. Only two sets of logical considerations ( $a$ and $\left.a^{\prime}\right)$ influence one's confidence in a specific conceptual hypothesis. Two additional considerations have the potential to dampen the confidence one has in the veracity of a theory about psychological events, and then two more considerations may dampen confidence in the veracity of a theory about the historical origins of those psychological events.

\section{Implications for Theories in Evolutionary Psychology}

It is with this logic of scientific persuasion in mind that we can appreciate why it is considerably harder for people to be persuaded about the veracity of some types of psychological theories compared to others. There are simply more obstacles to persuasion for theories with a historical model. These are not merely psychological obstacles that lie entirely within the heads of critics; rather, they are legitimate logical obstacles to the inference process. Some of these obstacles to persuasion apply across many different types of theories that specify models of the historical origins of psychological processes (including both evolutionary and sociocultural theories); other aspects apply more uniquely to evolutionary psychological theories.

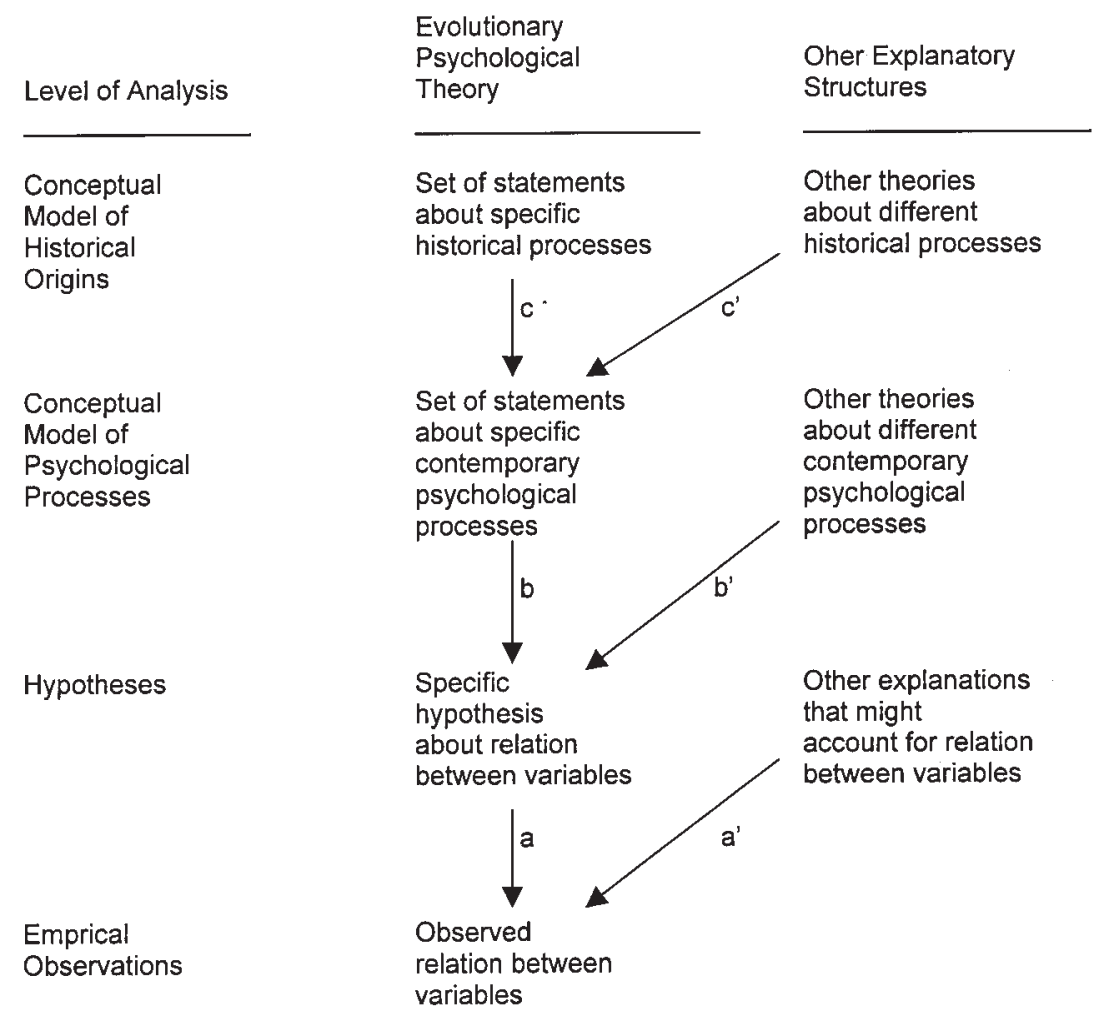

Figure 1. A schematic model of logical inference processes underlying scientific persuasion. 


\section{Inferential Obstacles Faced by All Theories Specifying Models of Historical Origin}

Multiple layers of inference. Consider again the example of gender differences in the preferred age of mates. Recall that the logic of falsification is such that if empirical evidence indicates no gender differences, then this evidence reflects equally negatively on all theories that predict such a difference-regardless of whether the theory specifies merely contemporary psychological processes or whether it specifies a historical model as well. However, what if the evidence reveals the predicted difference that men do indeed prefer younger women for mates, whereas women prefer older men. This positive evidence does not reflect equally on the different statements that logically imply this result. The more levels of inference that a predictive structure is removed from the results, the less persuasive those results are about the truth of that predictive structure. The likelihood is perhaps only modest that a person will confidently be persuaded that one particular model of psychological events must be correct. The likelihood is smaller still that a person will be persuaded that one particular model of historical origins must be correct. This example illustrates a fundamental point: Psychological theories specifying models of historical origins are, in general, more difficult to swallow whole than theories specifying only models of contemporary psychological events.

It is obvious from the model presented in Figure 1 that even if all levels of a historical theory maintain a relatively high batting average, the multiplicative effect of swallowing all three levels can still be quite low. As an illustrative example, suppose at every level of inference the logical consistency scores (represented by $a, b$, and $c$ ) are .7 and the probabilities associated with alternative explanations (represented by $a^{\prime}, b^{\prime}$, and $c^{\prime}$ ) are only .1. In spite of the persuasive probabilities that exist at of each level alone (e.g., $A=.7 *(1-.1)=.63$ ), the compound probabilities multiplied across all three levels is much lower $(C=.63 * .63 * .63=.25)$. Thus, even in the face of fairly convincing evidence predicted by the theory, the extent to which the whole theoretical edifice is perceived to be verified is still quite low.

This illustrates the difficulty faced by psychological theories that specify models of historical origin. Even if everything is generally logically compelling across the different levels of theory, it is difficult for scientists to feel confident that a result reflects the consequences of the historical processes specified by the theory.

Assumptions about historical circumstances.

Psychological theories have the logical structure of a set of "if-then" statements ("If $A$ then $B$," "If $B$ then $C$," "If $C$ then $D, E$, and $F$ ") that are deducible from some stipulated set of initial assumptions. A first step in judging the plausibility of a theory is an assessment of the assumptions on which it is based. If the assumptions are of questionable veracity, then the entire theoretical structure is likely to be judged implausible.

Theories describing contemporary psychological processes typically begin with assumptions that are easily observed and readily granted to be true. For example, no one really doubts the initial assumptions from which cognitive dissonance theory is derived (e.g., that individuals sometimes are subject to conflicting cognitions; Festinger, 1957). On the other hand, theories describing historical origins of psychological processes begin with assumptions about structures, events, or processes pertaining to the past (e.g., Cosmides, 1989; Eagly \& Wood, 1999; Greenberg et al., 1997). The past is less easily observed than the present and so the veracity of these assumptions is less readily granted. Therefore, compared to the logical plausibility accorded explanations based purely on contemporary psychological processes (symbolized by $b$ in the preceding equations), the logical plausibility accorded historical explanations (symbolized by $c$ ) is likely to be somewhat lower.

Alternative historical explanations. In addition, the perceived likelihood that alternative hypotheses or theories can explain a given result will almost certainly increase as one moves across the hypothetical, psychological, and historical levels (from $A$ to $B$ to $C$ in Figure 1). The confidence with which persons feel like they can rule out alternative hypotheses at the hypothesis-testing level $\left(a^{\prime}\right)$ is frequently quite high. At this level, the tools that aid people in ruling out alternative explanations (e.g., inferential statistics, experimental design) are relatively sophisticated. When applying this same logic at the level of psychological theories $\left(b^{\prime}\right)$ the task is more daunting. When scientists ask what models of psychological processes might yield a specific conceptual hypothesis, we often open a broad door through which many different theoretical structures can pass. Consequently $b^{\prime}$ is typically accorded a higher value than $a^{\prime}$.

Now consider theoretical structures that pertain to the historical past. Ruling out alternative explanations at the historical level $\left(c^{\prime}\right)$ is typically far more difficult than merely ruling out alternative explanations at the level of contemporary psychological events $\left(b^{\prime}\right)$. There are several reasons for this added difficulty. First, there is the issue of the temporal gap between cause and effect. Historical theories typically indicate some considerable passage of time between the proposed causal events (e.g., adaption pressures, the origins of social systems) and the psychological structures that those events are purported to give rise to. Thus, there is always plenty of time for other unidentified events or processes to give rise to the same structures. Second, there's the pragmatic difficulty of devising empirical methods that might actually comment on alternative 
explanations at the historical level (Conway, 1999; Lewontin, 1990; Richardson, 1996, 2000). It is difficult to employ psychological research methods to measure variables or processes purported to operate in the past. In the absence of empirical evidence, commentary on alternative explanations is limited to logical considerations alone (such as parsimony; see Archer, 1996, and Schmidt \& Buss, 2001 for examples).

Thus, not only do historical theories in psychology have to bear the weight of the cumulative impact of many layers of inference, they also have to deal with the fact that this last layer of inference-pertaining to models of historical origins-is by itself an exceedingly tough sell.

\section{Extra Inferential Obstacles Facing Evolutionary Psychological Theories}

Even among the small set of psychological theories that describe a model of historical origins, there is considerable variability. Some of these theories are more likely than others to arouse skepticism, suspicion, and charges of being unfalsifiable. Some, more than others, resist being swallowed whole. For instance, there are several distinct theories that describe historical origins of gender differences in sexual behavior-some with a strong evolutionary component (e.g., Buss \& Schmidt, 1993) and some without (e.g., Eagly \& Wood, 1999). It appears that people are more resistant to persuasion by evolutionary psychological theories than they are to other models of origins. Why is this? An answer demands attention to three dimensions along which evolutionary psychological theories typically differ from other theories of origins.

Temporal remoteness of specified origins. $\mathrm{Al}-$ though all historical theories suffer inferentially because they are set in a past that is no longer directly accessible, there is considerable variability in how far back in time these theories reach. Some models specify causal events that occurred in relatively recent history, whereas others specify events that occurred in the much more distant past. For instance, one prominent social roles theory of gender differences (Eagly, 1987; Eagly \& Wood, 1999) specifies that the origins of contemporary gender roles lie in historical events that are assumed to have occurred during the relatively brief and recent period of history since Homo sapiens appeared on the global scene. In contrast, theories specifying the evolutionary consequences of differential parental investment (Buss \& Schmidt, 1993; Gangestad \& Simpson, 2000) describe events that are assumed to have occurred during a more temporally remote epoch of history that predates the appearance of Homo sapiens. Thus, although both of these kinds of models are set in the past, the evolutionary model is more greatly removed from contemporary psychological processes.
This fact makes the linkage between the historical model and the contemporary model seem to be a much bigger and less straightforward deductive step. The difficulty in persuading individuals as to the veracity of models pertaining to the past is greater as those models dip more deeply into the past. The assumptions on which evolutionary models are based (biological characteristics of prehuman populations, the physical and social environments governing early hominid life, etc.), are based on inevitably spotty, inevitably imperfect clues about that very distant past (e.g., Tooby \& DeVore, 1987; Wrangham, 1987). In addition, the amount of time passed between a putative cause and an ostensible effect almost certainly serves as a heuristic indicating the likelihood that alternative historical theories can account for specified psychological structures. Because evolutionary models of origins are set so far in the past, there is much more time for other kinds of processes - such as sociocultural processesto have had an impact on our psychology (e.g., Scher, 1999; Wood \& Eagly, 2000).

In addition, as the temporal distance between origins and outcomes grows, it is less easy to convince skeptics that empirical evidence could possibly be collected that uniquely supports the specified link between historical and psychological structures. It is perhaps not unreasonable to entertain the hope that contemporarily available evidence (e.g., written histories or other cultural artifacts) might be found that reveals a hypothesized sequence of cultural events occurring within, say, the last 2 or 3 thousand years. It is a more farfetched hope that contemporarily available evidence might be found to reveal unambiguously a hypothesized sequence of evolutionary events occurring over the course of tens of thousands of years of human history. Moreover, one can relatively easily conceive of methodologies that, within a compressed contemporary time frame, might simulate the emergence of cultural norms over time (e.g., Arrow \& Burns, in press; Latané \& L'Herrou, 1996; Schaller \& Conway, 1999; Sherif, 1936). It is far more difficult to concoct contemporary methods that simulate- to any reasonable degree of fidelity - the natural and sexual selection processes hypothesized to underlie the emergence and evolution of human cognitive structures.

Complexity of the metatheoretical perspective. The more complex a metatheoretical perspective is perceived to be, the more likely that it will be perceived to yield multiple alternative explanations that might account for some observed phenomenon. All historical theories suffer inferentially because the historical past is complex. It is suspected, however, that evolutionary theories are especially likely to be affected by the perception of metatheoretical complexity.

The relation between biological adaptation processes and psychological processes is probably perceived by 
most people to be more complex than the relation between historical social structures and psychological processes. Evolutionary thinking suggests exceptionally complex processes - processes involving multifaceted relations between genes, phenotypic characteristics, and environments. Because the relation between phenotypic responses and genetic survival is complicated, it is no easy logical task to figure out the specific psychological traits that might have been adaptive in ancient environments (Caporael \& Brewer, 1995, 2000; Conway, 1999; Dawkins, 1976; Richardson, 2000). The same adaptive problem may be solved in multiple different ways; responses to very different adaptive pressures may result in very similar phenotypic consequences. This complexity confers uncertainty; working within the evolutionary metatheoretical framework, theorists may find multiple logically distinct ways of accounting for a given phenomenon.

Even when no specific alternative theories are readily available, the complexity of the relation between behaviors, genes, and survival may leave people with a vague feeling of mystery and uncertainty. This may heuristically compel a subjective sense that other historical (evolutionary or otherwise) theories might also yield similar predictions - even if one cannot imagine exactly what those theories might be. Thus, even without clear challengers to evolutionary theories, people may yet still be uniquely skeptical of the ability to rule out alternative theoretical explanations for these theories.

Relative size of history model within the theoretical framework. A third dimension of importance refers to the relative size of the historical model within the context of the relevant theory (see Schaller \& Conway, 2000). Some theories that include historical models articulate those models briefly as points of departure and devote the bulk of theoretical statements to the articulation of contemporary psychological events (e.g., Eagly, 1987; Solomon et al., 1991). The relative size of the historical model is small. In contrast, within the framework of most evolutionary psychological theories, the historical model is much more than a mere point of departure. Indeed, the presence of a well-specified historical model of origins is required for that theory to be considered to fall within the domain of evolutionary psychology. For this reason, within evolutionary theories of psychology, the relative size of the historical model is frequently large.

Relative size matters. As we have shown, it is comparatively more difficult to be persuaded as to the veracity of a model of historical origins (Formula 3, previously) than it is to be persuaded as to the veracity of a model of psychological events (Formula 2). Therefore, the bigger the relative size of the historical model within a larger theoretical structure, the more difficult it is to be persuaded as to the truth of that whole theory.

\section{Where Do We Go From Here?}

This analysis helps to explain why theories in evolutionary psychology are commonly viewed with greater skepticism than more traditional psychological theories. These considerations, coupled with the fact that it is essential to be intellectually persuasive to succeed in the scientific environment, might tempt the pragmatic scientist to play it safe-to avoid dabbling in multilevel theories that specify models of historical origins.

Giving in to this temptation, however, would surely have unhealthy consequences for the advancement of psychological science. There are already plenty of pressures on scientists to think small (Garcia, 1981; Horgan, 1996; Hull, 1988). Although there may be shortterm personal advantages of a narrow epistemological approach (e.g., one's own ideas are less likely to be maligned as wrong, and one can more confidently trust others' ideas as well), these are offset by a considerable scientific downside (e.g., vanishingly small inferential returns on empirical investment and stagnation of theory development). There is plenty of concern that there is an increasing tendency toward this sort of epistemological conservatism in psychology (Higgins, 1992; Kruglanski, 2001; Schaller \& Crandall, 1998; Wallach \& Wallach, 1998); it's important to fight off the easy temptation to play it safe. Thinking big and working with ambitious multilevel theories helps ensure a healthier, more progressive science (Kenrick \& Simpson, 1997; Solomon et al., 1991).

The constructive conclusion to draw from our analysis is this: Scientists need to continue to develop ambitious multilevel theories like those in evolutionary psychology, but we need to do it better. The logical and psychological considerations identified previously imply several practical guidelines toward articulating evolutionary psychological theories that are maximally persuasive.

\section{Rigorous Specification of a Model of Historical Events}

As logical structures, scientific theories comprise sets of statements that follow from some initial set of assertions. In the case of evolutionary psychological theories, the assertions that form the conceptual starting point pertain to constraints operating on populations long ago. The consequences of those constraints - specified in a model of historical events - occurred over a considerable stretch of time. If a theory is to be perceived as plausible, these initial assertions and the model of historical events must be rigorously and believably specified.

Because they pertain to circumstances existing in the unobservable past, the believability of the assertions underlying evolutionary psychological theories 
rests on intuition or inference or both. Intuition alone allows people to readily accept the supposition that the physiological differences currently describing men and women must have existed for a very long time. Consequently, the considerable archeological and biological evidence supporting that supposition is scarcely required to be convinced that differential parental investment must have described immense chunks of human history. (Given its immediate intuitive appeal, it is no surprise that this assertion has offered the starting point for so much work in the realm of evolutionary psychology.) Not all assertions about the constraints operating on populations can be intuited with such confidence, however. Assertions about social structures within ancient populations cannot be based on intuition alone. Any theory or model proceeding from an assertion about social structures during ancient epochs is unlikely to be fully persuasive unless intuition is supplemented with rigorous documentation attesting to the plausibility of those assertions. Audiences need to be apprised of relevant and credible anthropological and zoological evidence before they can be expected to accept any assertion about the social structures of ancient populations.

Documenting these initial assertions is merely the first step in articulating a plausible model of historical events. The next step is the rigorous specification of the evolutionary consequences of the asserted constraints. Many applications of evolutionary thinking to psychology skimp on this step-indicating merely that "Given constraint $X$, it makes sense that adaptive consequence $Y$ would have occurred." That may be true, but it is not logically convincing. Specific constraints never necessarily require some specific adaptive consequence; any of several other consequences might have been equally adaptive. The failure to attend to the multiple plausible consequences of any single constraint can lead to the statement of a theory that-although logically falsifiable - may be easily dismissed. More compelling theories include more fully articulated descriptions of the multiple constraints operating simultaneously on human populations (e.g., social constraints as well as constraints posed by existing anatomical structures) and more carefully explain why some particular adaptive consequence was more likely to emerge than other possible consequences.

To this end, it is important for evolutionary psychologists to do thorough cost-benefit analyses to be maximally persuasive. Historically, evolutionary scientists in general and evolutionary psychologists in particular have had a tendency to attend almost exclusively to potential benefits of a proposed adaptive trait while ignoring potential costs (Gangestad \& Simpson, 2000). Failure to engage in full cost-benefit analyses has at least two negative consequences for evolutionary psychologists. First, because it is frequently easy for persons to imagine such costs for supposedly adaptive traits (see, e.g., Conway, 1999), the skeptic may dismiss a theory as soon as any unidentified cost of the hypothesized adaptation comes to mind. Thus, incorporating potential costs of an adaptation into an evolutionary theory does not allow the skeptic this "easy out" and forces criticisms to be more fully articulated. Secondly, not considering both costs and benefits of traits also forfeits the possibility of deducing more complex and novel hypotheses (see Gangestad \& Simpson, 2000). All seemingly adaptive traits have potential costs, and analysis of those costs is probably more likely to lead down surprising and novel pathways (more on this following).

\section{Rigorous Specification of Model of Psychological Events}

Rigorous specification of the historical model needs to be accompanied by even more rigorous specification of a model of contemporary psychological events. Sloppy theories may skip breezily from descriptions of ancient evolutionary environments to predictions about contemporary variables. To be persuasive, a theory needs to specify the contemporary psychological processes that are assumed to have resulted from the ancient operations of natural selection, and that in turn yield those predictions.

At minimum, it is necessary to specify the model of contemporary psychological events as distinct from the model of historical (evolutionary) events. Logically, the two models operate at different levels of analysis and specify relations between very different classes of variables. (Among other things, evolutionary models describe processes pertaining to populations, whereas psychological models describe questions pertaining to individuals.) Among audiences inexperienced in differentiating between these levels of analysis, the relatively unverifiable statements about ancient evolutionary processes may be lumped together with the more immediately verifiable statements about contemporary psychological processes. This may lead to a false skepticism concerning the model of psychological processes. Clearer separation between the two levels of analysis increases the perceived verifiability of the model of contemporary psychological events.

If indeed the model of contemporary psychological events is perceived to have merit, then it is more likely that the model of historical events will be perceived to have merit as well. The inferential distance from empirical observation to the acceptance of the evolutionary model is rarely spanned in a single leap; it is more readily traversed through the two more cautious logical steps identified in Figure 1. In fact, acceptance of the more proximal model may often be necessary before psychological audiences are willing to consider acceptance of the evolutionary model. Thus, the articulation of a more coherent and com- 
plete psychological model will have the effect of increasing the perceived plausibility of that historical model as well. Moreover, any increase in the treatment of the psychological model diminishes the relative size of the historical model within the theoretical whole and thus decreases the relative proportion of the theory that is perceived to be unverifiable.

\section{Explicit Articulation of Deductive Logic}

Scientists often make deductive leaps intuitively and do not necessarily experience those leaps as exercises in logic. Consequently, a hypothesis that is deduced from a set of preliminary assertions may be expressed simply as an intuitively plausible prediction but not as the end product of deductive reasoning. Within some theoretical contexts, this approach may work just fine. However, within the context of evolutionary psychology - in which the inferential distance from initial assertion to hypothesis is perceptually huge-this approach may be problematic. Evolutionary psychological theories are more convincing if presented within a narrative that clearly reveals the underlying deductive logic.

There are several reasons for this. First, a coherent deductive narrative reveals that an evolutionary theory is truly a theory about psychological processes and is not merely a post hoc explanation. This is a fundamental distinction. Theories are "forward-thinking" entities. They comprise sets of statements that are connected logically as the result of being deduced from the same preliminary assertions. On the other hand, explanations are "backward-thinking" entities. They comprise sets of statements that are connected psychologically as a result of the attempt to explain the origins of some specific observed phenomenon. People intuitively engage in both forward- and backward-thinking processes. Individual scientists may seek theories for the pragmatic purpose of finding explanations, and therefore backward-thinking explanatory processes naturally play a role in discoveries of forward-thinking theories. Nevertheless, the logic of a theory exists separately from the means through which it was discovered and from the phenomena for which it provides an explanation. That logical distinction is not always evident in the manner in which a theory is articulated, however. If theorists do not present an explicit account of the deductive logic that forms the guts of any good theory, others may (with good reason) be more likely to assume that the theory is simply a convenient backward-thinking explanation of a single phenomenon or set of phenomena. On the other hand, if theorists explicitly lay out the deductive logic that underlies the theory, others will be more likely to perceive the theory as something that exists separate from a set of phenomena that they theory may (or may not) have originally been developed to explain. This articulation of the deductive logic of a theory helps a theory to appear-and to be-more forward thinking.

Second, careful articulation of deductive logic enhances the perceived explanatory coherence of a theory (Thagard, 1992). The perceived coherence of the theory suffers whenever links between different elements of the theory are unclear. Any slippage or mystery in the connection between the historical and contemporary levels of analysis (symbolized by $c$ in Figure 1) undermines the persuasiveness of the historical model. Any perceived looseness in the manner in which hypotheses are generated also maintains skepticism. Convincing evolutionary psychological theories require careful articulation of the deductive logic through which a model of psychological events is deduced and through which hypotheses testing this model are generated.

Consider the following example. Suppose we start with the intuitively appealing assumption that in ancient environments it would have been adaptive if we helped those genetically related to us (i.e., our kin) but less adaptive if we helped nonkin. Ignoring our own advice-for the sake of expediency - to engage in a full cost-benefit analysis, let's say we loosely base this assumption on the idea that, from a gene survival viewpoint, we potentially have a lot to gain by ensuring that our kin survive and nothing directly to gain by ensuring that our nonkin survive. In addition, suppose we deduce from this assumption that, in contemporary times, we should help our kin more than our nonkin.

This sounds reasonable on the surface, but it's sloppy. It leaves out all the steps between the ancient past and the psychological present. For example, it does not articulate exactly what psychological processes would have been involved in the adaptation. This failure can have negative consequences: The skeptic may reasonably argue that people do not frequently think about genetic relatedness in an explicit way, and the skeptic may dismiss the theory on these grounds. This sloppy deduction also does not articulate how those psychological processes might influence contemporary helping behavior. This, too, can have negative consequences: The skeptic may think the theory suggests that people rarely or never help nonkin and may readily produce many instances where people do help those with no genetic relationship. Again, the skeptic may dismiss the theory-quite reasonably—on these grounds.

Articulating the deductive logic more explicitly makes such easy outs more difficult to come by. For example, let's start again with the assumption that helping kin, from an adaptationist perspective, is more adaptive than helping nonkin. Therefore, one could argue, it would have been adaptive to distinguish between kin and nonkin in a quick and efficient way through the use of salient kinship cues. These cues would trigger psychological responses (emotions, cog- 
nitions) that compel quick behavioral helping responses. In contemporary times, these psychological processes (sensitivity to kinship cues, linkage between perception of cues and emotion or cognition) remain and influence contemporary helping behavior when (and only when) those cues are present.

Articulating the preceding outlined steps makes it clearer what specific predictions the theory does and does not make. It thus does not allow the skeptic to dismiss the theory on, say, the grounds that people don't explicitly think very much about genetic relatedness-because the newly articulated deduction makes it clear that they are not expected to. As a result, it is far more compelling than the original, sloppy version devoid of such specific linkages. Thus, it is to evolutionary psychologists' advantage to present as much of the deductive logic that attempts to link ancient environments to contemporary processes as possible. As we have shown, the logical and psychological gap between the two is enormous, and evolutionary psychologists do not have very much room with which to be sloppy.

\section{Generation of Truly Novel Psychological Implications}

Rigorous deductive logic not only strengthens the internal coherence of a theory but also provides an important means of generating novel hypotheses. This is not only essential to a progressive program of scientific research (Lakatos, 1970), it also is one of the primary means through which theories prove to be persuasive. Indeed, the generation of novel hypotheses has been the focus of statements attesting to the merit of evolutionary psychological theories (Buss et al., 1998), as well as statements expressing concerns about their utility $(\mathrm{Ca}-$ porael \& Brewer, 2000; Forster \& Shapiro, 2000). It seems clear that people are unlikely to be excited by evolutionary psychological theories that appear merely to explain previously observed phenomena or to offer alternative conceptual structures within which to locate previously articulated hypotheses. People will be more compelled by evolutionary psychological theories that yield novel hypotheses specifying previously undocumented psychological phenomena.

Of course, conceptual novelty is more a matter of uniqueness than temporal precedence. Logically, it matters not at all who first articulates a hypothesis if, in fact, it turns out that the same hypothesis can also be deduced from alternative theoretical frameworks. The evolutionary theories that are most likely to be persuasive are those that yield hypotheses that cannot be deduced from other theories and that remain uniquely deducible over time. Hypotheses like these are rarely arrived at through intuitive means alone. This suggests that the greatest intellectual dividends offered by evolutionary psychology will follow from the adoption of an inquisitive "let's see where this takes us" approach: Start with a plausible assertion about some set of evolutionary constraints and consider the various consequences that follow logically from that assertion. In doing so, it is valuable to ignore easy temptations to arrive at previously discovered or intuitively obvious destinations and instead to try to find routes to subtle, surprising, previously unchartered psychological territory.

Conceptually novel hypotheses are rarely simple. Complex interaction effects involving three or more variables are almost always less obvious than mere main effects of one variable on another. Also, the more complex the interaction, the less easy it is to identify alternative explanations for the effect (Conway, Schaller, Tweed, \& Hallett, in press). Among the evolutionary psychological theories that are most useful and convincing to skeptics are those that yield complex hypothesis specifying not only how people (and subsets of people) think, but also the specific domains within which those psychological processes do and do not proceed and the specific contexts that trigger those domain-specific psychological processes. One example is provided by evolutionary psychological work on the domain- and situation-specificity of human reasoning processes: On the basis of the assumption that, in ancient environments, it would have been adaptive to detect social "cheaters" (that is, persons who violated implicit social contracts), it was deduced that persons would reason differently in cheater-detection situations than in situations not relevant to social contract violations. Tests of these proposed domain-specific reasoning abilities have supported this notion (e.g., Cosmides, 1989; but see Pollard, 1990). Other evolutionary psychological theories yielding similarly complex hypotheses are emerging in the areas of social cognition and interpersonal behavior (Gangestad \& Simpson, 2000; Kurzban \& Leary, 2001; Neuberg \& Cottrell, in press; Schaller, in press). Within the contemporary psychological environment, convincing tests of theories typically depend on the deduction of and empirical support for these sorts of complex and twoand three-way interactions. Thus, when attempting to uncover and articulate theories in evolutionary psychology, it will become increasingly important to articulate theories that not only predict general human tendencies but also predict specific domains and situational contexts that moderate (amplify or inhibit) those tendencies.

\section{Forge Conceptual Integrations Between Alternative Models of Psychological Events}

Psychologically and logically, a theory is less persuasive under conditions in which a different theory yields the same hypotheses. There is a logical caveat to this alternative explanations rule, however: To the de- 
gree that the alternative theoretical explanation is logically compatible with the specified theory, this should lessen the negative impact of that alternative explanation on the perceived veracity of the theory. Indeed, psychological phenomena are almost always multiply determined, and it is rare that alternative explanations identify incompatible psychological processes. Thus, although legitimate alternative explanations will always reduce the perceived veracity of a theory, at a purely logical level this ought to be moderated to some degree by the logical compatibility of the theories under the microscope.

This point is especially relevant when considering evolutionary psychological theories and the nonevolutionary processes that are often offered as alternative explanations for phenomena predicted by evolutionary theories. Many of these alternative explanations specify psychological processes that are logically independent of the processes specified by evolutionary psychological models. For instance, evolutionary psychological theories concerning gender differences in sexual behavior typically specify (or imply) the existence of some contemporary physiological basis for observed gender differences. Plausible alternative explanations typically focus on the existence of some contemporary cultural norms as the basis for observed gender differences. Obviously, there is nothing incompatible about the coexistence of physiological and cultural structures. Nor is the alternative explanation-the existence of specific cultural norms-incompatible with any model of evolutionary events precipitated by the constraints of differential parental investment. In fact, the existence of gender-relevant cultural norms can actually be deduced logically from the same evolutionary starting point (Schaller, 1997). This is not uncommon. Many hypothesized evolutionary pressures relevant to psychology could logically have led simultaneously to the emergence of specific physiological structures and to the emergence of specific social structures that reify or amplify or both the operation of those physiological structures (Boyd \& Richerson, 1985; Dunbar, Knight, \& Power, 1999; Durham, 1991; Janicki \& Krebs, 1997; Lumsden \& Wilson, 1981; Tomasello, 1999; Tooby \& Cosmides, 1992).

The implication for evolutionary psychological theorists is this: By pursuing multiple deductive paths that follow from initial assertions, it is possible to articulate internally coherent theories describing the historical emergence and contemporary operation of different sets of psychological processes. By linking multiple models of psychological processes to the same historical origins, it is possible not only to decrease the number of ostensible alternative explanations that bear negatively on the persuasiveness of an evolutionary theory but to incorporate those explanations into the theory so that they bear positively instead (see also Simpson \& Gangestad, 2001). The result, if successful, will be a theory that has greater explanatory coherence (Thagard, 1992) and so is all the more persuasive.

Attempts to do this not only serve evolutionary psychological theories, but also- by providing conceptual integrations of superficially unrelated psychological processes-provide important benefits to the psychological sciences in general.

\section{Concluding Remarks}

To be allowed to tread on the schoolyard of scientific discourse, a theory has to meet certain minimal criteria such as falsifiability. Once admitted through that gate, however, these minimal criteria matter very little to the ultimate success of that theory. Survival on the harsh playground of scientific skepticism depends on many deeper features that contribute to the theory's utility, as judged by scientists. Scientists judge a theory to be useful to the extent that they perceive it to be true. Scientists are people, and people judge a theory to be true to the extent that it is perceived to be verifiable. However important it is psychologically, though, verifiability maintains little presence in the positivist philosophy of science that most psychological scientists are taught to follow. It is not surprising, therefore, that inferential doubts based on verifiability are expressed instead in the culturally acceptable language of falsifiability.

If these doubts are taken literally, they are easily dismissed, and it is all too common for enthusiasts of evolutionary psychology to shrug off criticisms expressed in the language of falsifiability - to attribute these criticisms to ignorance or enmity or both. To do so, however, is to miss an opportunity for critical reflection. Lurking within these misdirected criticisms is a substantial truth: For reasons both logical and psychological, theories within evolutionary psychology are different from other sorts of theories in psychology. They are not different in kind but in degree: the degree to which they are verifiable and thus the degree to which they are intuitively persuasive. The upshot is that evolutionary psychological theories must be crafted more carefully, articulated more precisely, and tested more rigorously if they are to overcome the skepticism that naturally greets them. Individual scientists have a responsibility to these theories, as we all do to all youthful but promising theoretical approaches within our field. When responding negatively to evolutionary psychological theories, scientists have a responsibility to criticize them thoughtfully, to identify legitimate flaws. When responding positively to evolutionary psychological theories, scientists have a responsibility to attend to those flaws as well, to improve on them. Through careful intellectual parenting, these promising theories may survive long enough to contribute substantially and persuasively to our understanding of contemporary human psychological processes. 


\section{References}

Archer, J. (1996). Sex differences in social behavior: Are the social role and evolutionary explanations compatible? American Psychologist, 51, 909-917.

Arrow, H., \& Burns, K. L. (in press). Self-organizing culture: How norms emerge in small groups. In M. Schaller \& C. S. Crandall (Eds.), The psychological foundations of culture. Mahwah, NJ: Lawrence Erlbaum Associates, Inc.

Bechtel, W. (1988). Philosophy of science: An overview for cognitive science. Hillsdale, NJ: Lawrence Erlbaum Associates, Inc.

Bem, D. J. (1972). Self-perception theory. In L. Berkowitz (Ed.), Advances in experimental social psychology (Vol. 6, pp. 1-62). New York: Academic.

Boyd, R., \& Richerson, P. J. (1985). Culture and the evolutionary process. Chicago: University of Chicago Press.

Buss, D. M. (1989). Sex differences in human mate preferences: Evolutionary hypotheses tested in 37 cultures. Behavioral and Brain Sciences, 12, 1-49.

Buss, D. M., Haselton, M. G., Shackelford, T. K., Bleske, A. L., \& Wakefield, J. C. (1998). Adaptations, exaptations, and spandrels. American Psychologist, 53, 533-548.

Buss, D. M., \& Schmidt, D. P. (1993). Sexual strategies theory: An evolutionary perspective on human mating. Psychological Review, 100, 204-232.

Caporael, L. R., \& Brewer, M. B. (1995). Hierarchical evolutionary theory: There is an alternative, and it's not creationism. Psychological Inquiry, 6, 31-34.

Caporael, L. R., \& Brewer, M. B. (2000). Metatheories, evolution, and psychology: Once more with feeling. Psychological Inquiry, 11, 23-26.

Conway, L. G., III. (1999). Noise, logic, and the span of time. American Psychologist, 54, 440-441.

Conway, L. G., III, Schaller, M., Tweed, R. G., \& Hallett, D. (2001). The complexity of thinking across cultures: Interactions between culture and situational context. Social Cognition, 19, 228-250.

Cosmides, L. (1989). The logic of social exchange: Has natural selection shaped how humans reason? Studies with the Wason selection task. Cognition, 31, 187-276.

Crandall, C. S., \& Schaller, M. (in press). Scientists and science: How individuals' values shape collective norms. In M. Schaller \& C. S. Crandall (Eds.), The psychological foundations of culture. Mahwah, NJ: Lawrence Erlbaum Associates, Inc.

Dawkins, R. (1976). The Selfish gene. New York: Oxford University Press.

Dunbar, R., Knight, C., \& Power, C. (1999). The evolution of culture. Edinburgh, Scotland: Edinburgh University Press.

Durham, W. H. (1991). Coevolution: Genes, culture, and human diversity. Stanford, CA: Stanford University Press.

Eagly, A. H. (1987). Sex differences in social behavior: A social-role interpretation. Hillsdale, NJ: Lawrence Erlbaum Associates, Inc.

Eagly, A. H., \& Wood, W. (1999). The origins of sex differences in human behavior: Evolved dispositions versus social roles. American Psychologist, 54, 408-423.

Festinger, L. (1957). A theory of cognitive dissonance. Evanston, IL: Row, Peterson.

Forster, M. R., \& Shapiro, L . A. (2000). Prediction and accommodation in evolutionary psychology. Psychological Inquiry, 11, 31-33.

Gangestad, S. W., \& Simpson, J. A. (2000). The evolution of human mating: Trade-offs and strategic pluralism. Behavioral and Brain Sciences, 23, 573-644.

Garcia, J. (1981). Tilting and the windmills of academe. American Psychologist, 36, 149-158.

Gigerenzer, G. (1998). Ecological intelligence: An adaptation for frequencies. In D. D. Cummins \& C. Allen (Eds.), The evolution of mind (pp. 9-29). New York: Oxford University Press.
Gould, S. J. (1997, June 26). Evolution: The pleasures of pluralism. New York Review of Books, 44, 47-52.

Greenberg, J., Solomon, S., \& Pyszczynski, T. (1997). Terror management theory of self-esteem and cultural worldviews: Empirical assessments and conceptual refinements. Advances in Experimental Social Psychology, 29, 61-139.

Greenwald, A. G., \& Ronis, D. L. (1981). On the conceptual disconfirmation of theories. Personality and Social Psychology Bulletin, 7, 131-137.

Higgins, E. T. (1992). Increasingly complex but less interesting articles: Scientific progress or regulatory problem? Personality and Social Psychology Bulletin, 18, 489-492.

Horgan, J. (1996). The end of science. New York: Broadway Books.

Hull, D. L. (1988). Science as a process. Chicago: University of Chicago Press.

Janicki, M. G., \& Krebs, D. L. (1997). Evolutionary approaches to culture. In. C. Crawford \& D. L. Krebs (Eds.), Handbook of evolutionary psychology (pp. 163-207). Mahwah, NJ: Lawrence Erlbaum Associates, Inc.

Kelley, H. H. (1967). Attribution theory in social psychology. In D. Levine (Ed.), Nebraska Symposium on Motivation (Vol. 15, pp. 192-238). Lincoln: University of Nebraska Press.

Kenrick, D. T., \& Keefe, R. C. (1992). Age preferences in mates reflect sex differences in mating strategies. Behavioral and Brain Sciences, 15, 75-91.

Kenrick, D. T., \& Simpson, J. A. (1997). Why social psychology and evolutionary psychology need one another. In J. A. Simpson \& D. T. Kenrick (Eds.), Evolutionary social psychology (pp. 1-20). Mahwah, NJ: Lawrence Erlbaum Associates, Inc.

Ketelaar, T., \& Ellis, B. J. (2000). Are evolutionary explanations unfalsifiable? Evolutionary psychology and the Lakatosian philosophy of science. Psychological Inquiry, 11, 1-21.

Kitcher, P. (1985). Vaulting ambition: Sociobiology and the quest for human nature. Cambridge, MA: MIT Press.

Kitcher, P. (1993). The advancement of science. New York: Oxford University Press.

Kruglanski, A. W. (1994). The social-cognitive bases of scientific knowledge. In W. R. Shadish \& S. Fuller (Eds.), The social psychology of science (pp. 197-213). New York: Guilford.

Kruglanski, A. W. (2001). That "vision thing": The state of theory in social and personality psychology at the edge of the new millennium. Journal of Personality and Social Psychology, 80, 871-875.

Kunda, Z., \& Thagard, P. (1996). Forming impressions from stereotypes, traits, and behaviors: A parallel-constraint-satisfaction theory. Psychological Review, 103, 284-308.

Kurzban, R., \& Leary, M. R. (2001). Evolutionary origins of stigmatization: The functions of social exclusion. Psychological Bulletin, 127, 187-208.

Lakatos, I. (1970). Falsification and the methodology of scientific research programmes. In I. Lakatos \& A. Musgrave (Eds.), Criticism and the growth of knowledge (pp. 91-196). Cambridge, England: Cambridge University Press.

Latané, B., \& L'Herrou, T. (1996). Spatial clustering in the conformity game: Dynamic social impact in electronic groups. Journal of Personality and Social Psychology, 70, 1218-1230.

Laudan, L. (1977). Progress and its problems. Berkeley, CA: University of California Press.

Lewontin, R. C. (1990). The evolution of cognition. In D. N. Osherson \& E. E. Smith (Eds.), An invitation to cognitive science: Thinking (pp. 229-246). Cambridge, MA: MIT Press.

Lumsden, C. J., \& Wilson, E. O. (1981). Genes, mind, and culture: The coevolutionary process. Cambridge, MA: Harvard University Press.

Merton, R. K. (1942). Science and technology in a democratic order. Journal of Legal and Political Sociology, 1, 115-126. 
Neuberg, S. L., \& Cottrell, C. A. (in press). Intergroup emotions: A biocultural approach. In D. M. Mackie \& E. R. Smith (Eds.), From prejudice to intergroup relations: Differentiated reactions to social groups. Philadelphia: Psychology Press.

Peters, R. H. (1976). Tautology in evolution and ecology. American Naturalist, 110, 1-12.

Pollard, P. (1990). Natural selection for the selection task: Limits to social exchange theory. Cognition, 36, 195-204.

Popper, K. R. (1959). The logic of scientific discovery. New York: Hutchison Education.

Quine, W. (1953). From a logical point of view. Cambridge, MA: Harvard University Press.

Richardson, R. C. (1996). The prospects for an evolutionary psychology: Human language and human reasoning. Minds and Machines, 6, 541-547.

Richardson, R. C. (2000). Epicycles and explanations in evolutionary psychology. Psychological Inquiry, 11, 46-49.

Schaller, M. (1997). Beyond "competing," beyond "compatible." American Psychologist, 52, 1379-1380.

Schaller, M. (in press). Ancestral environments and "motivated" social perception: Goal-like blasts from the evolutionary past. In S. Spencer \& S. Fein (Eds.), Motivated social cognition: The Ninth Ontario Symposium. Mahwah, NJ: Lawrence Erlbaum Associates, Inc.

Schaller, M., \& Conway, L. G., III. (1999). Influence of impression-management goals on the emerging contents of group stereotypes: Support for a social-evolutionary process. Personality and Social Psychology Bulletin, 25, 819-833.

Schaller, M., \& Conway, L. G., III. (2000). The illusion of unfalsifiability and why it matters. Psychological Inquiry, 11, 49-52.

Schaller, M., \& Crandall, C. S. (1998). On the purposes served by psychological research and its critics. Theory and Psychology, 8, 205-212.

Scher, S. J. (1999). Are adaptations necessarily genetic? American Psychologist, 54, 436-437.
Schmidt, D. P., \& Buss, D. M. (2001). Human mate poaching: Tactics and temptations for infiltrating existing mateships. Journal of Personality and Social Psychology, 80, 894-917.

Sherif, M.(1936). The psychology of social norms. New York: Harper.

Simpson, J. A., \& Gangestad, S. W. (2001). Evolution and relationships: A call for integration. Personal Relationships, 8, 341-355.

Solomon, S., Greenberg, J., \& Pyszczynski, T. (1991). A terror management theory of social behavior: The psychological functions of self-esteem and cultural worldviews. Advances in Experimental Social Psychology, 24, 93-159.

Thagard, P. (1992). Conceptual revolutions. Princeton, NJ: Princeton University Press.

Tomasello, M. (1999). The human adaptation for culture. Annual Review of Anthropology, 28, 509-529.

Tooby, J., \& Cosmides, L. (1992). The psychological foundations of culture. In J. Barkow, L. Cosmides, \& J. Tooby (Eds.), The adaptive mind: Evolutionary psychology and the generation of culture (pp. 19-136). New York: Oxford University Press.

Tooby, J., \& DeVore, I. (1987). The reconstruction of hominid behavioral evolution through strategic modeling. In W. G. Kinzey (Ed.), The evolution of human behavior: Primate models (pp. 183-237). Albany, NY: State University of New York Press.

Wallach, M. A., \& Wallach, L. (1998). When experiments serve little purpose: Misguided research in mainstream psychology. Theory and Psychology, 8, 183-194.

Wood, W., \& Eagley, A. H. (2000). A call to recognize the bredth of evolutionary perspectives: Sociocultural theories and evolutionary psychology. Psychological Inquiry, 11, 52-55.

Wrangham, R. W. (1987). The significance of African apes for reconstructing human social evolution. In W. G. Kinzey (Ed.), The evolution of human behavior: Primate models (pp. 51-71). Albany, NY: State University of New York Press. 